\title{
Developing a dedicated cestode life cycle database: lessons from the hymenolepidids
}

\author{
F. LEFEBVRE ${ }^{1}$, B. B. GEORGIEV ${ }^{2}$, R. A. BRAY ${ }^{3}$, D. T. J. LITTLEWOOD ${ }^{3}$
}

\author{
${ }^{1}$ Independent researcher, 47 rue des 3 Rois, 86000 Poitiers, France; scientific associate with The Natural History \\ Museum, London, UK, E-mail: f.lefebvre@nhm.ac.uk; ${ }^{2}$ Central Laboratory of General Ecology, Bulgarian Academy \\ of Sciences, 2 Gagarin Street, 1113 Sofia, Bulgaria, E-mail: bbg@ecolab.bas.bg; ${ }^{3}$ The Natural History Museum, \\ Department of Zoology, Cromwell Road, London SW7 5BD, UK, E-mails: rab@nhm.ac.uk,t.littlewood@nhm.ac.uk
}

\begin{abstract}
Summary
The Cestode Life Cycle Database (CLCdb) project was initiated in 2005 with the objective to develop a comprehensive and centralised resource to store, retrieve and analyse key information concerning tapeworm life cycles; e.g. morphogenesis, intermediate host identities, transmission patterns, etc. It constitutes the first electronic database to deal with complex life cycle information for any helminth taxon. Here we critically evaluate our experience after exhaustively entering data for our model group, the cyclophyllidean family Hymenolepididae. After providing basic statistics (530 consulted references; 230 'known' life cycle), we identify future needs in turning the CLCdb into an open access monograph covering all cestode groups. We review the added benefits and potential utilities of the database for cestodologists and other users, including ecologists and veterinarians, and we call for specialist contributions. Since late 2007, a short version of the CLCdb has been available online, with basic functionalities and tools (www.nhm.ac.uk/research-curation/projects /cestode-life-cycle/index.html).
\end{abstract}

Keywords: tapeworms; monograph; e-resource; larval biology; biodiversity

\section{Introduction}

Electronic databases are now established as invaluable tools for biological research (see e.g. Soberón \& Peterson, 2004). In their simplest form, on an individual desktop computer, they constitute a centralised resource to store information structured in a way that allows some flexibility for development, and commonly with tools that allow userspecified, targeted interrogation of the contents. When online, their potential applications and utilities are again broadened, not least through integration with other webbased resources. Using the internet as the medium of data access and exchange, databases become dynamic and in- teractive with the possibilities of simultaneous contributions and regular updates. Indeed, even when comprehensive paper monographs/reviews do exist, they can become out of date almost before they are published as new species are discovered, classifications are revised and geographical distribution data are expanded.

As proclaimed by Janzen (2004), 'now is the time' to apply any and all relevant modern tools in traditional zoology to revitalise the entire field and to capture the wealth of over 150 years of comparative information. The databasing of parasite life cycles is particularly timely because the number of morphologists able to identify larval stages (from published literature or slide preparations) is dramatically declining. Their expertise is however invaluable not only for taxonomic identification but also in helping to interpret and translate specialist knowledge into a same and universal language of science (i.e., English-based). A move towards a centralised and comprehensive database is also timely because experimental life cycle studies are getting rarer every year. The golden age of such studies was in the 1970 - 1980's and, although compiling available data is not a race against time, we cannot currently assess the state of accumulated knowledge, utilise it properly or be aware of the extent of our ignorance. The now common use of information technologies (internet media, public access databases, digital libraries, etc.) provides the opportunity for the involvement of all stakeholders and the development of dedicated tools for sharing all available information, all with just a handful of initial contributors. The Cestode Life Cycle Database project (hereafter CLCdb) was initiated in 2005 with the aim of providing a centralised and comprehensive open resource covering all key available information on larval tapeworm biology; e.g. morphogenesis, intermediate host identity, transmission process, etc. The CLCdb initiative was carried out along with other existing and complementary databases in mind, namely: 1. The Global Cestode Database (GCD: 
www.cestodedatabase.org; principal investigators: Janine Caira, Kirsten Jensen and Claire Healy). This on-going project aims to generate an authoritative list of all nominal species in cestodes, and to provide revised taxonomic treatments along with on-line access to original descriptions. It is a systematic and taxonomic resource mainly concentrating on adult cestode data. 2. The Tissue Database (principal investigators: Peter Olson and Tim Littlewood) is an on-going depository for organising the collection of tissues for gene sequencing, along with providing biological details of the collected specimens and hosts. It covers most parasitic helminth groups and includes data on both the adult and larval stages. This collection falls under the auspices of the NHM's collection and will eventually be databased as part of the NHM's commitment to make details of its collection available via the Internet. Currently, the Tissue Database is managed separately and locally (NHM, London). 3. The HostParasite Database (H-Pdb: www.nhm.ac.uk/researchcuration/projects/host-parasites; principal investigators: David Gibson, Rod Bray and Eileen Harris) is an electronic resource with details on host-parasite associations recorded from the scientific literature over the period 1988-2003. It covers all helminth parasites and is fully cross-referenced with in excess of 28,000 publications in over a quarter of a million records. The database complements catalogued paper records held by the Parasitic Worms Group (NHM, London) dating from 1922. To our knowledge, the CLCdb constitutes the first electronic database to deal with complex life cycle information for any helminth taxon (although there exist published paper monographs for digeneans and nematodes of vertebrates, i.e. Yamaguti, 1975 and Anderson, 2000, respectively). Here, we make the first review on the advancement of the project, after exhaustively entering data for our model group, the cyclophyllidean family Hymenolepididae. We critically evaluate the applied methodology, take lessons from our experience and identify future needs in turning the CLCdb into an open access monograph covering all cestode groups.

\section{Material and Methods}

We adopted a systemic approach, and began by populating the database with life cycle information concerning the family Hymenolepididae Ariola, 1899. We chose to start with this taxon mainly because resolved life cycles were expected to be relatively abundant (see estimates in Beveridge, 2001) and because one of us (BBG) has some taxonomic experience with the group. Hymenolepidids form the most species-rich family in cestodes with $\sim 1000$ nominal species described to date (our computation, based on previous indexing such as Schmidt, 1986 and McLaughin, 2003), thus constituting a real and challenging model system to start with.

As for any database project, the work up to completion may be visualised in a series of discrete steps, we can conveniently adapt to our case. We thus identified four major steps in the databasing process of life cycle data:

1. Find data: literature search. Knowledge of life cycles has been sparse and is generally distributed throughout disparate publications in numerous languages spanning over 150 years. We started the literature search by consulting available reviews and/or monographs for subgroups of hymenolepidids (e.g., Spasskaya, 1966; Genov, 1984; Maksimova, 1989; Bondarenko \& Kontrimavichus, 2006). From these, we compiled an initial list of primary references for each species, and then copied/ordered them using the NHM library facilities. Having collected a first set of paper copies, we extended the list with additional references given as citations therein (in general those cited in the introduction) and proceeded as such, working iteratively up to the primary (pioneering) publications concerning each species. As a complement, we searched the bibliographic database Zoological Record (covering literature from 1864 to present; Thomson Scientific, Inc.), using hymenolepidid species names so far recorded, to identify backwards references that may have been omitted, and forwards new life cycle studies arising since the last reviews and/or monographs. Finally, the online version of the Host-Parasite Database was searched for more hostparasite associations and references. This database, although of limited time coverage (1988 - 2003), was compiled by working specialists in helminthology and as such may include potentially useful information from more obscure sources (e.g., conference proceedings and other non-referenced reports).

2. Organise data: database design. The CLCdb was created in the application FileMaker Pro 8 (FileMaker, Inc.) and consists of one master table (Life Cycle table) and two related satellite tables (Reference table and Host table). The Reference table functions as an internal bibliographic manager (such as EndNote or Thomson Reuters) to help tracking, ordering, storing and easily retrieving the sources of primary information from which data are to be extracted. It contains all necessary fields to record any type of bibliographic references (and also museum collection voucher - specimens, although none are entered yet), along with other functionalities. In particular, we introduced a 'key words' field, in which each cestode species under investigation in a given reference may be associated with pre-defined identifiers that encapsulate the nature of the data available (e.g., egg/embryo morphology, egg/embryo transmission, metacestode morphology, metacestode host, metacestode transmission, adult morphology, adult host). By doing so, we know exactly the content of each reference in our library. The Life Cycle table was designed to record data on the infection process, taxonomy and morphology of the developmental stages (i.e., egg/embryo, metacestode(s), adult). The table contains more than 200 fields to be able to record the developmental sequence of the most complex life cycles (up to four hosts). The Host table is used in parallel to record additional information about the host of any cestode developmental stages (e.g., 
host taxonomy, host habitat).

3. Enter data: speaking the same language. As life cycles may be diverse and complex, elements to describe and record them need to be standardised. To ensure consistency, we devised a number of rules for entering life cycle data into the CLCdb. These included: 1. Data entry is reference-based throughout, so that every bit of entered information can be linked to its primary source, and associated with details of the databaser (mainly FL and BBG so far). 2. Information is recorded as given in the primary source (i.e., terminology and taxonomy employed by the authors). Taxonomy of both cestode and host species are to be revised later in consultation with monographs and/or group specialists. The only two exceptions concern the 'metacestode name' and the 'host status'. These two fields need to be recorded with the reference paper at hand as they may refer to drawings or non-explicitly written information, and in accordance with standard definitions (as given in the Glossary; see the CLCdb web site). 3. For any given developmental stage, information is recorded about the most advanced stage whenever possible (i.e., metacestode infective for the next host; sexually mature adult). 4 . In the case of natural infections, use one record per cestode developmental stage and per host. In contrast, enter the full sequence within a single record when the developmental chronology has been experimentally demonstrated. In these cases, use as many records as there is 'experimentally shown' succession of hosts.

4. Check data: taxonomic revision. As hosts and parasites names were entered as they appeared in the reference sources, taxonomy has to be checked by group specialists to conform to current standards. For hymenolepidids, the (re-)attribution of larval descriptions to valid species was the responsibility of one of us (BBG). The taxonomic revision was based on the classification adopted for the project Fauna Europaea (www.faunaeur.org), i.e., following Spasskaya (1966) for hymenolepidids of birds and Czaplinski and Vaucher (1994) for hymenolepidids of mammals, adopting also some subsequent developments. Host taxonomy is to be revised and updated in accordance with current authorities using recently published monographs or online databases. Nevertheless, a comprehensive check of all host names is beyond the scope of the project.

\section{Results}

\section{Overall content}

At the time of this first published update, the CLCdb contains 3,500 records in the Reference table, 1,970 records in the Life Cycle table and 3,550 host-parasite associations in the Host table, corresponding to $\sim 600$ cestode taxa (original species names without corrections for synonymy or inconsistent spelling). However, the dataset is yet only exhaustive and fully revised for the hymenolepidids $(\sim 400$ taxa, see section below and Table 1). Additional recorded taxa correspond to those life cycle data from other groups and published in the same papers along with hymenolepidid data (predominantly for the order Cyclophyllidea).

The CLCdb now has a presence on the web, with a preliminary version made available within the NHM domain in December 2007 (www.nhm.ac.uk/research-curation/ projects/cestode-life-cycle). So far, however, only the Reference table, the Host table and the Glossary have been uploaded. This first iteration provides a glimpse of the data we expect to be able to serve from this portal in the near future.

\section{The hymenolepidids case}

After taxonomic revision, life cycle data were attributed to over 230 taxa (see Table 1), including 215 valid species, plus 13 species incertae sedis, and some unidentified/unnamed species (given as Genus sp.). Actual knowledge for these species is up to the point of knowing the metacestode morphology ( 8 recognised variants of the cysticercoid type; see Chervy, 2002) and at least one identified intermediate host. In hymenolepidids, the life cycle involved a single invertebrate intermediate host (excluding paratenic snail hosts) and a definitive host (with the exception of Rodentolepis nana for which the whole life cycle can occur within the mammalian definitive host). Detailed results will be provided in a forthcoming article reviewing the known diversity of life cycles in hymenolepidids (Lefebvre et al., in prep.).

Table 1. Content of the CLCdb (June 2008). Data exhaustive and fully revised only for the hymenolepidids

\begin{tabular}{lcc}
\hline & Hymenolepididae & Cestoda \\
\hline Nb references & 530 & 3500 \\
Nb records & 2190 & 3550 \\
Nb taxa & 400 & 600 \\
Nb species & 230 & - \\
\hline
\end{tabular}

Life cycle data in hymenolepidids were extracted from 530 primary references (mostly journal articles), although much more were actually consulted (with no relevant information or non-original data). Overall, existing life cycle knowledge for a given species was collected from an average of five references. However, as illustrated in Fig. 1 , the frequency distribution is highly skewed to the right (best fit by a negative binomial distribution) with overdispersed data (median value: 2 references). For more than half of the species (138 out of 230), all available knowledge was encapsulated in not more than one or two references ( 93 species with a single source of information). In contrast, 10 species had their data dispersed over 405 references (82 for Hymenolepis diminuta, 62 for Sobolevicanthus gracilis, 44 for Fimbriaria fasciolaris). In other terms, about $5 \%$ of the species necessitated consultation of $80 \%$ of the total number of references. Similarly, the frequency distribution of the number of identified intermediate hosts per species is highly skewed to the right (see 


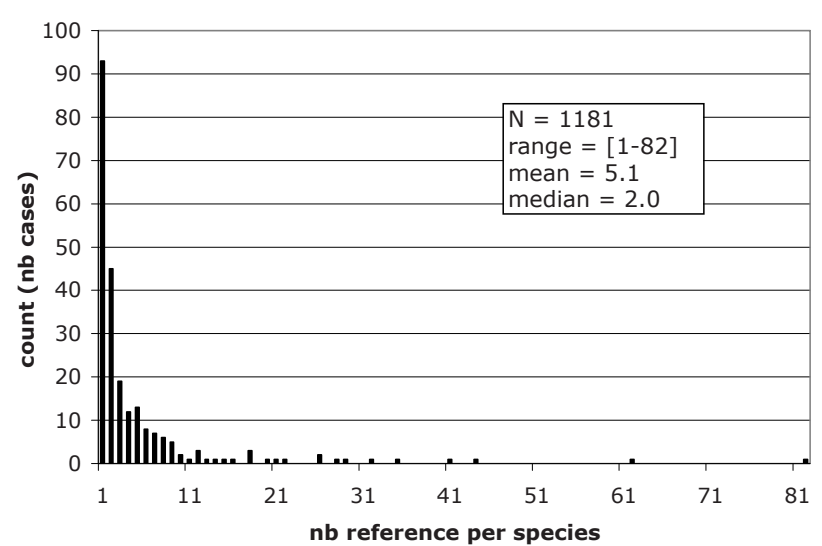

Fig. 1. Frequency distribution of the number of reference(s) used to extract all available life cycle data per species in the family Hymenolepididae

Fig. 2). For more than half of the species (142 out of 230), the number of recorded intermediate hosts is very limited (less than 3) while quite a few species exhibit a low specificity (median: 2; extreme value: 55 ). The relationship between the number of identified intermediate hosts and the number of references consulted in order to gather this information is particularly informative. Although there is a positive and significant relationship between the two variables ( $\mathrm{Rs}=+0.74, \mathrm{~N}=233, \mathrm{p}<0.001)$, the slope of the regression line is $<1$ (after Ln transformation, intercept set to $0: \mathrm{y}=0.73 * \mathrm{x}$; see Fig. 3). In other words, across all species, the trend is for less than one recorded intermediate host per reference, which may reveal saturation in the information content gained from additional sources. This is best illustrated with the case of Hymenolepis diminuta, for which $32 \%$ of its references ( 26 out of 82 ) consisted of routine experiments using either Tribolium confusum or Tenebrio molitor. The yet applied methodology thus involved a large research effort for a handful of wellstudied species, while gathering many redundant data.

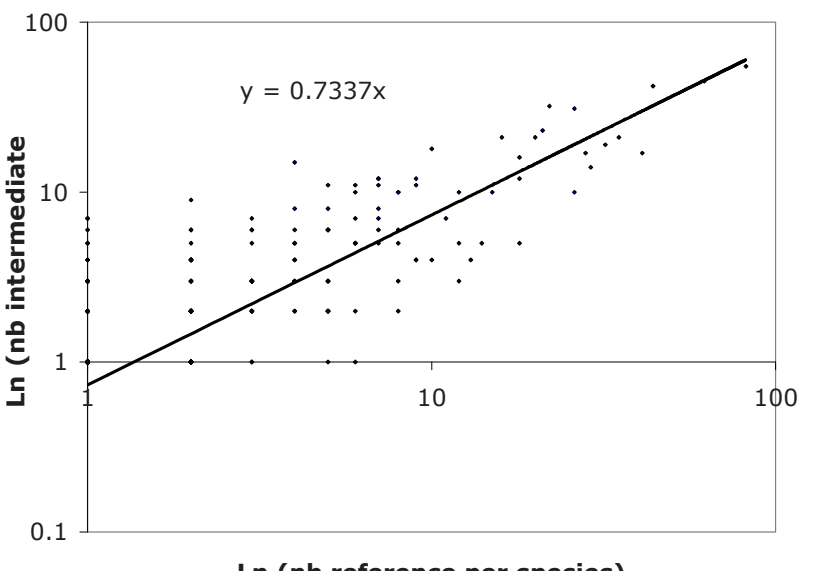

Fig. 3. Relationship between the number of reference(s) and the number of recorded intermediate host(s) per species in the family Hymenolepididae (after Ln transformation of both variables, and forced intercept to 0 )

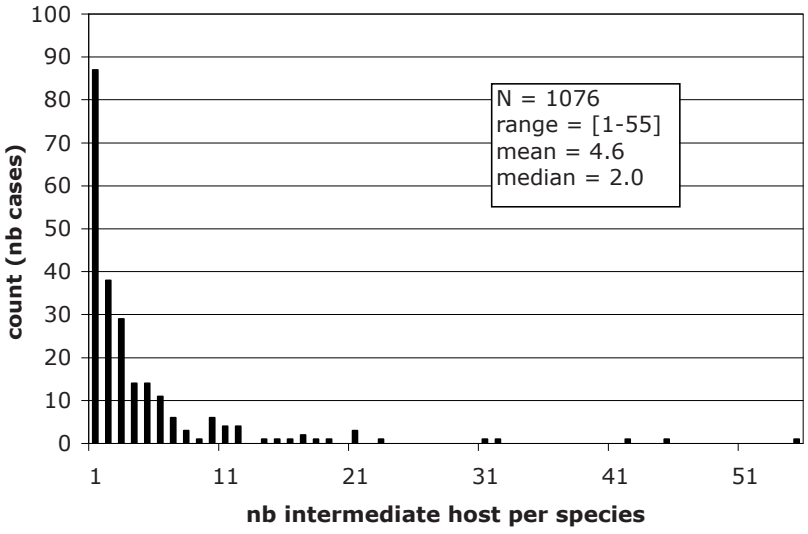

Fig. 2. Frequency distribution of the number of recorded intermediate host(s) per species in the family Hymenolepididae

\section{Discussion}

According to previous estimates (Beveridge, 2001), life cycles in the family Hymenolepididae were known for about 49 species ('up to the point of morphogenesis'). With some restrictions (e.g., concerning the understanding of a 'known' life cycle), our methodical and exhaustive search has thus revealed a better knowledge than previously thought concerning hymenolepidid life cycles ( $\sim$ 4-fold increase). Keeping this ratio in mind and using other estimates given by Beveridge (2001) for the whole cestode group (i.e., 220 species with known life cycles), one can reasonably envisage that partial information concerning life cycles might be available in the literature for approximately 800-900 cestode species.

We plan to record all this available knowledge and turn the $\mathrm{CLCdb}$ into an open-access resource, functioning as an online monograph with species pages. In achieving this objective, we need to critically evaluate the initial experience with our model system, establish priorities and strategies, and secure additional funding.

\section{Critical evaluation}

Here is a list of conveniences and practical problems we encountered over each phase of the database process, together with the specific responses we eventually adopted: 1. Find data. The identification and collection of primary references was greatly simplified thanks to the NHM facilities (i.e., access to multiple bibliographic databases, reprint collection of the Parasitic Worm Group, large and long-term collection of world wide journals in the main library, ordering possibilities through inter-library exchanges, efficient and dedicated library staff). Actually, less than 10 identified references have yet to be collected for the whole hymenolepidids (mainly from Japanese journals and Russian book series), thus culminating in the most complete literature collection of life cycle studies (for the Hymenolepididae, and probably for the Cestoda).

2. Organise data. The primary sources of information are believed to be conveniently and efficiently managed. Col- 
lected paper copies were physically stored and organised (alphabetical order of the first author) in one dedicated life cycle bank/library, consultable on request (CLC cabinet, Parasitic Worms Group, The Natural History Museum, London). The bibliographic details were entered in the Reference table of the CLCdb and, with a keyword field and other tools, this internal bibliographic manager is fully functional, and has already proved to be very useful.

We experienced some difficulties in creating a functional and normalised database. This was clearly due to an initial lack of knowledge in structuring a relational database, particularly as fields were added or developed during the initial phases. We also experienced numerous problems with making effective the online version of the database. Most of this was due to NHM institutional policies but also related to the lack of normalisation in the internal structure of the database. This problem has since been addressed with the help of a FileMaker consultant, and we now have the solution to soon move towards a high profile relational database with greater functionality.

3. Enter data. Effective input of the database requires an awareness of the underlying biology of cestodes. Perhaps most difficult to overcome has been the proliferation of confusing and idiosyncratic terminology - something that has been highlighted during various International Cestode Workshops and partly solved through the combined efforts of the user community (see, for instance, Chervy, 2002 for metacestode morphotypes). For the purposes of the CLCdb, the problem was addressed by consulting cestodologists and creating a dedicated Glossary setting up the definition of $\sim 150$ terms related to life cycle and cestode biology. Also, as nearly half of the life cycle literature had been published in local/national journals, data entry into the CLCdb necessitated regular translation into English. In addition to the skills of some of us (especially BBG for Cyrillic-based Slavic languages), this was achieved with the occasional help of foreign scientists, volunteers or students visiting the NHM in London.

4. Check data. Cestode taxonomy has to be validated by international authorities able to (re-)attribute larval descriptions to current valid species. This may be the most critical part of the project. For hymenolepidids, this was achieved by one of us (BBG), and the cestode community agreed during the last International Workshop (Smolenice, Slovakia, 2008) on a list of leading specialists for taxonomic revision in each cestode groups (at the family level for the cyclophyllideans, and at the ordinal level for the remaining cestodes). The taxonomy of the hosts has been partly updated using a couple of online databases (i.e., Fauna Europaea, WoRMS and ITIS, in that order of priority). Moreover, we initiated a deeper check in asking our NHM colleagues in the different departments to provide us with the reference details of what they consider the 'best' monographs/resources for their group(s) of expertise. This was successfully applied for gathering gross biology of each hymenolepidid intermediate hosts, and since hosts are in general better known than their parasites, there is no reason to think that this procedure could not be extended to cover the remaining host taxa.

Finally, it is fair to admit that the CLCdb, like any other compilation initiative at this stage, may contain a substantial amount of erroneous data. Failures may come from inconsistencies introduced by the databaser (e.g., typographic errors), from misunderstanding of the text and/or illustration (especially in the case of older literature, in non-English languages) or from erroneous data given by the author(s) of the studies (e.g., imprecise description, wrong taxonomic identification). Eventually, life cycle information could be checked by reviewing accumulated data periodically and using statistics to identify outliers (i.e., extreme points in term of size or host range can be easily pin-pointed out this way), as done in hymenolepidids for the need of a forthcoming article on life cycle diversity (Lefebvre et al., in prep.).

\section{Next steps and improvements}

1. We plan to involve the whole cestode community to cover life cycle knowledge for the remaining cestodes. However, rather than taking an exhaustive approach, as done with the hymenolepidids, we aim to be selective. First, when dealing with primary literature, no more than 3 - 4 major references could be selected per species. For most taxa, the problem is solved de facto with the paucity of life cycle knowledge (in general, one or two references are available per species). For well-studied species, group specialists could help in identifying the most important contributions to begin with; for instance, this might include the oldest investigation (for historic purpose), the most detailed (in terms of description, host taxonomic range, etc.) and the most recent synthesis (for an update list of previous works). Secondly, we consider an exemplar approach to be valid, whereby we target key constituent species representing a taxonomic group and encapsulating the diversity of it constituent tapeworm life cycles (in terms of metacestode morphology, host taxonomic range, number of intermediate hosts, ways of transmission). This choice may also be dictated by targeting taxa used in the molecular phylogenies, because of the analytic perspectives it may offer, with the objective of securing another funding (see point 3 ).

2. There is a need to improve both versions of the CLCdb. The desktop computer version has to be partly remodelled with the creation of new tables to manage the link between the original and the revised taxonomy. Additional normalisation is also required; for instance, a better use of ID fields (foreign and primary keys) to relate the different tables and to minimise the amount of redundant data. Although the present version is functional and workable for its current use, this is a crucial element to maintain high performance as the database content increases and also in the perspective of joining other existing databases. The online version first needs to allow full access to the exact content of the desktop version, in uploading the Life Cycle table. The Web site would also benefit the addition of advanced functionalities, such as 'species pages' (summarising in a single layout all available knowledge so 
far recorded as multiple individual records) and 'tree search' (for exploring the actual content of data using a hierarchical taxonomic key) to turn the online database into a fully interactive, open-access, monograph on cestode life cycles.

3. Additional funding is required. To secure this, we need to promote the database and demonstrate its usefulness (see below for possible applications). Utility of the constituent data and the database as a tool are indeed the key factors for its continued growth and development. Globalisation, climate change, urbanisation, transfer of species, modification of diet and habitat are all likely to affect the emergence or re-emergence of helminth-mediated diseases, and it is well established how knowledge of parasite life cycles aids in developing means of preventing transmission. A full understanding of biomedically important helminths requires understanding of host transmission, at least those known (or potentially expected) as natural reservoirs (intermediate or definitive hosts) that may not be targeted currently by treatment programmes focused on treating humans or livestock. Also, researchers engaged in biodiversity research need to become involved. Parasites as indicators of ecosystem function and health as well as underlying complexity (through inferences made concerning the trophic connections dictated by tapeworm life cycles) are considered worthy of future research. As Wheeler (2004) noted, 'biodiversity assessment necessitates research tools and projects on new scales and a cultural change among taxonomists who need now to function as a community'. The CLCdb project, along with other on-going databases, falls under the scope of these two major schemes (biodiversity assessment and help in diseases control) for cestode parasites initially.

\section{Current utilities and potential applications}

1. The primary and currently available utility of the CLCdb is to generate lists of host-parasite associations for cestode larval stages (and adults when involved in experiments dealing with metacestode). One can be interested in having a list of known intermediate hosts for a given cestode species or, alternatively, a list of cestode species for a given host, along with reference to primary literature from which the information is extracted. Using such search tools, it may then be quick and easy to identify the likely vectors of a tapeworm infection in a given area.

2. From the database, we can generate customised 'on demand' reports for quantitative and qualitative studies in evolutionary ecology. In particular, one of our primary interests in the project was to generate matrices of characters that can be mapped onto available phylogenies to enlighten the evolution of life history at various taxonomic levels (see, for instance, Cribb et al., 2003 for digeneans). Also, large datasets can be conveniently exported to develop cladistic hypotheses (see, e.g., Hoberg et al., 2000 in taeniids) or to test macro-evolutionary hypotheses to explain the observed (or non-observed) diversity and distribution of groups of organisms (review in Morand and Poulin, 2003). Finally, as raw data in the CLCdb are based on individual references, exports can be used in metaanalyses to reveal general trends and patterns over multiple independent studies.

3. The CLCdb could also be used as a diagnostic tool to help identifying larval stages entering the taxonomy of the host in which it was found and a set of morphological characters (size range, number and length of the hooks, etc.); e.g., a search in the database for cestode species known to infect Artemia sp. and having 10 rostellar hooks of a length less than $20 \mu \mathrm{m}$ returns 2 matching records: Hymenolepis californicus and Wardium fusa. Results can be then refined using known geographical distribution or other ecological details before reference to original taxonomic descriptions (using bibliographic details provided along with the search). This is an interesting tool for non-specialists that may help supplement (but never replace) the decline in taxonomic expertise.

4. Finally, one promising use of the CLCdb would be to develop a predictive model able to generate some clues for as yet unknown life cycles. The rationale is as follows: using comparative data from species with known life cycles (CLCdb) plus interrelationships between species (inferred from independent molecular and/or morphological characters) and assuming that closely related species share more biological features than distant ones (phylogenetic signal), one can target the most likely life cycle characters (e.g., intermediate host taxonomy) for any cestode species with yet unresolved life cycle. Moreover, considering the phylogenetic distances to the closest species with known life cycles (e.g., number of nodes in a cladogram), and including a measure of the quality of the closest known life cycles (e.g., number of independent studies), one can provide a confidence value around a predicted life cycle. Also, life cycle prediction could be further refined with the minimum biological data accompanying any species description (e.g., host habitat, host diet, egg structure and shape). Such a predictive tool would never replace actual verification (using helminthological surveys and/or experimental infections) but may enable more rapid reactions and more efficiency in the diagnosis of emerging/reemerging diseases.

\section{Acknowledgements}

We thank Serge Morand and Ian Beveridge for their invaluable contributions during the initial phase of the project (database design, field definitions, cestode terminology). We are very grateful to Aneta Nowodworska, Anna Lindsey-Clark, Guilhem Rascalou, Catherine Gray and Michael Hardman for their help in 'feeding' the database. We acknowledge Mike Sadka and Julia Howlett (IT Department) for their contribution in setting up the online version of the database within the NHM domain. We are also grateful to the staff from the NHM libraries, and particularly Angela Thresher, for their regular help in dealing with obscure references. This work was funded by NERC (NER/A/S/2003/00313). 


\section{References}

ANDERSON, R. C. (2000): Nematode parasites of vertebrates: their development and transmission. CABI publishing, Wallingford

BEVERIDGE, I. (2001): The use of life-cycle characters in studies of the evolution of cestodes. In LiTTLEWOOD, D. T. J. AND BRAY, R. A. (Eds): Interrelationships of the Platyhelminthes. Taylor and Francis, London

BONDARENKO, S., KONTRIMAVICHUS, V. (2006): [Aploparaksidae of wild and domesticated birds]. In Movsesyan, S. O. (Ed): Fundamentals of Cestodology, 14. Nauka, Moscow

CHERVY, L. (2002): The terminology of larval cestodes or metacestodes. Syst. Parasitol., 52: 1 - 33

CRIBB, T. H., Bray, R. A., Olson, P. D., LitTlewood, D. T. J. (2003): Life cycle evolution in the Digenea: a new perspective from phylogeny. Adv. Parasitol., 54: $197-254$ Czaplinski, B., VAuCHer, C. (1994): Family Hymenolepididae Ariola, 1899. In KHALIL, L. F., JONES, A. AND BRAY, R. A. (Eds): Keys to the cestode parasites of vertebrates. $\mathrm{CAB}$ International, Wallingford

GENOV, T. (1984): [Helminths of insectivores and rodents in Bulgaria]. Izdatelstvo na Balgarskata Akademiya na Naukite, Sofia

Hoberg, E. P., Jones, A., Rausch, R. L., Eom, K. S., GARDNER, S. L. (2000): A phylogenetic hypothesis for species of the genus Taenia (Eucestoda: Taeniidae). $J$. Parasitol., 86: 89 - 98

Janzen, D. H. (2004): Now is the time. Philos. Trans. $R$. Soc. B, 359: $731-732$

MCLaughlin, J. D. (2003): An annotated checklist of hymenolepidid cestodes described from birds: 1983-2002, Parassitologia, 45: 33 - 45

MAKsimova, A. P. (1989): [Hymenolepidid cestodes of the water fowl of Kazakhstan]. Akademiya Nauk Kazakhskoi SSR, Alma-Ata

Morand, S., Poulin, R. (2003): Phylogenies, the comparative method and parasite evolutionary ecology. Adv. Parasitol., 54: $281-302$

SCHMIDT, G. D. (1986): Handbook of tapeworm identification. CRC Press, Inc., Boca Raton

Soberón, J., PETERSON, A. T. (2004): Biodiversity informatics: managing and applying primary biodiversity data. Philos. Trans. R. Soc. B, 359: 689 - 698

SPASSKAYA, L. P. (1966): [Cestoda of birds in the USSR: Hymenolepididae]. Izdatel'stvo Nauka, Moskva

WHEELER, Q. D. (2004): Taxonomic triage and the poverty of phylogeny. Philos. Trans. R. Soc., B, 359: 571 - 583

YAMAGUTI, S. (1975): A synoptical review of life histories of digenetic trematodes of vertebrates with special reference to the morphology of their larval forms. Keigaku Publishing Co., Kyoto 